\title{
Laboreal
}

Volume $10 \mathrm{~N}^{\circ} 1$ | 2014

Análise ergonómica do trabalho e formação

\section{Restricciones organizativas, distorsión de la comunicación y sufrimiento ético: el caso de los centros de atención telefónica}

Constrangimentos organizacionais, distorção da comunicação e sofrimento ético : o caso dos centros de atendimento telefónico

Contraintes organisationnelles, distorsion de la communication et souffrance éthique : le cas des centres d'appels téléphoniques

Organizational constraints, distorted communication and ethical suffering: the case of call centers

\section{Duarte Rolo}

\section{OpenEdition}

Journals

\section{Edição electrónica}

URL: http://journals.openedition.org/laboreal/5420

DOI: $10.4000 /$ laboreal. 5420

ISSN: 1646-5237

\section{Editora}

Universidade do Porto

\section{Refêrencia eletrónica}

Duarte Rolo, «Restricciones organizativas, distorsión de la comunicación y sufrimiento ético: el caso de los centros de atención telefónica », Laboreal [Online], Volume $10 N^{0} 1$ | 2014, posto online no dia 01 julho 2014, consultado o 24 setembro 2020. URL : http://journals.openedition.org/laboreal/5420 DOI : https://doi.org/10.4000/laboreal.5420

Este documento foi criado de forma automática no dia 24 setembro 2020.

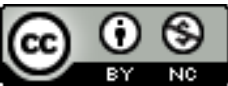

Laboreal está licenciado com uma Licença Creative Commons - Atribuição-NãoComercial 4.0 Internacional. 


\title{
Restricciones organizativas,
} distorsión de la comunicación y sufrimiento ético: el caso de los centros de atención telefónica

\author{
Constrangimentos organizacionais, distorção da comunicação e sofrimento \\ ético : o caso dos centros de atendimento telefónico \\ Contraintes organisationnelles, distorsion de la communication et souffrance \\ éthique : le cas des centres d'appels téléphoniques \\ Organizational constraints, distorted communication and ethical suffering: the \\ case of call centers
}

\section{Duarte Rolo}

\section{REFERÊNCIA}

Rolo, D. (2013). Contraintes organisationnelles, distorsion de la communication et souffrance éthique. Thèse de doctorat, Conservatoire National des Arts et Métiers, Paris.

\section{NOTA DO EDITOR}

http://dx.doi.org/10.15667/laborealx0114dres

Manuscrito recibido en: Marzo/2014

Aceptado tras peritaje: Mayo /2014

1 La tesis que aquí se presenta tiene como punto de partida la pregunta acerca del impacto psicológico de algunas formas específicas de la organización del trabajo, a 
saber, un conjunto de restricciones que impulsan el uso de la mentira, que se convierte en un elemento clave de la actividad de los trabajadores. La descripción clínica de los elementos relativos a las formas de organización del trabajo y la manera en que estas formas son experimentadas por los trabajadores fue uno de los principales objetivos de la tesis. En este contexto, se realizó un estudio de caso en un sector profesional específico: los centros de atención telefónica o call centers. La recolección del material empírico se realizó de acuerdo a los principios metodológicos de la psicodinámica del trabajo, que constituye el marco teórico que sustenta los debates celebrados a lo largo de la tesis.

\section{Analizar el trabajo desde la perspectiva de la psicodinámica}

2 Heredera de los estudios pioneros de la psicopatología del trabajo y de la ergonomía de la actividad (Wisner, 1995), la psicodinámica del trabajo se dedica al análisis y comprensión de los procesos intersubjetivos generados por la experiencia laboral. Entre las diferentes disciplinas que se interesan al trabajo actualmente, la psicodinámica del trabajo otorga un papel central a la interacción entre el sujeto y su actividad específica para tratar de entender el surgimiento de nuevas formas de sufrimiento en el trabajo. La confrontación entre el hombre y el trabajo real - es decir, todo aquello que resiste al control y al dominio de conocimientos técnicos o científicos - implica, invariablemente, una forma de desestabilización. Por lo tanto, la confrontación con lo real siempre se traduce, en primer lugar, en una experiencia desagradable. Lo real se revela en forma de accidente o avería, y nos impone de una forma o de otra un fracaso. Así, la confrontación con lo real es principalmente una experiencia afectiva. La psicodinámica optó por dar a esta experiencia el nombre de sufrimiento. El sufrimiento no es solamente una consecuencia negativa del trabajo. Más bien, es la que lleva al empleado a buscar una solución que le permitirá superar su propio sufrimiento que lo persigue. Por lo tanto, este sufrimiento no significa necesariamente una experiencia patológica. Bajo ciertas condiciones, el mismo sufrimiento puede llegar a ser un motor para la creatividad.

3 Teniendo en cuenta estos elementos, la comprensión de la experiencia subjetiva de los trabajadores se centra en las manifestaciones de sufrimiento en el trabajo. Por eso los métodos de investigación se centran en la dimensión subjetiva del trabajo, recogida a través de la observación formulada por los sujetos acerca de su propia actividad. Por consiguiente, en esta área de estudio los instrumentos de recolección de datos son invariablemente cualitativos.

En nuestro caso, se utilizaron tres métodos de acceso al trabajo real de los operadores de centros de llamadas:

- En primer lugar, aún en una fase exploratoria, hemos desarrollado una serie de entrevistas individuales no directivas con los trabajadores de centros de atención telefónica;

- Luego, un período de observación participante en una plataforma telefónica;

- - Por último, se realizó una investigación colectiva en conformidad con los principios de la psicodinámica del trabajo (Ganem, 2011) [1]. 


\section{El trabajo en centros de atención telefónica y la mentira prescrita}

4 Los elementos recogidos a partir del trabajo con los telemarketers revelan que la mentira se ha convertido en un aspecto central de su actividad. Por mentir se debe entender cualquier acto en el que un hombre enuncia como verdadero algo que sin embargo es falso .Dicho de otra manera, mentir es comunicar intencionalmente a los demás una visión de la realidad diferente de la que consideramos verdadera. En el caso estudiado, la prescripción para mentir es consecuencia de una combinación de factores que llevan los operadores a tomar la mentira como modo de funcionamiento. En general, no existe una orden explícita o directa para mentir. Sin embargo, los agentes de telemercadeo son conscientes de que los objetivos que se les imponen no se pueden lograr sin mentir (los objetivos de ventas definidos son imposibles de alcanzar sin mentir a los clientes, por ejemplo).En consecuencia, aunque no forma parte explícita de la tarea, la mentira se vuelve una dimensión fundamental de la actividad.

5 De hecho, la investigación realizada revela que la mentira sirve, en la mayoría de los casos, como una palanca comercial. Su uso diario refuerza una retórica cuyo objetivo es aumentar las ventas a toda costa. Incluso si eso significa truco y engañar a los clientes. Tales prácticas no son, finalmente, inofensivas. Muchos trabajadores se muestran molestos con este tipo de prácticas que contradicen sus principios. El hecho de que la mentira se haya infiltrado en la actividad puede ser problemático para la salud mental, siendo que no siempre es posible conciliar este tipo de limitación con una ética de trabajo que respete los valores del servicio. De hecho, esta contradicción no siempre es fácil de soportar. El sufrimiento experimentado por los trabajadores en estas circunstancias se encuentra estrechamente relacionado con el conflicto entre sus propios valores y los valores que subyacen a las prácticas que estipula la organización del trabajo

\section{Conflicto de valores y sufrimiento ético}

6 Estos cargos implican la renuncia a un conjunto de valores que hasta entonces habían guiado la conducta profesional de la mayoría de los teleoperadores. La adopción de medidas o la ejecución de órdenes que, al final de cuentas, desaprueban, pueden generar una forma específica de sufrimiento que la psicodinámica del trabajo a apodado "sufrimiento ético" (Dejours, 1998). Surge el sufrimiento ético cuando el sujeto llega al punto en que ejecuta órdenes con las que no está de acuerdo. Servir a un sistema que el propio trabajador rechaza brinda lugar a un conflicto entre aquello que la persona sabe que no debería aceptar, pero que aun así hace. Al contradecir sus valores, los trabajadores corren el riesgo de perder la consideración que tienen de sí mismos.

7 Pero la traición de creencias o ideales no da necesariamente lugar a una angustia moral o a un sentimiento de culpa. Incluso no es cierto que constituya sistemáticamente la causa de enfermedades o trastornos psicológicos. Varios estudios realizados en clínica del trabajo demuestran efectivamente que el "trabajo sucio" (Hughes, 2010) es compatible con la realización personal. Entonces, ¿Cómo podemos explicar que algunos de los trabajadores entrevistados desarrollen síntomas relacionados a los conflictos 
laborales que enfrentan, mientras que otros no dan signos significativos de aflicción? Es imposible dar una respuesta satisfactoria a esta pregunta sin recurrir a uno de los principales descubrimientos de la psicodinámica del trabajo: las estrategias de defensa contra el sufrimiento en el trabajo.

\section{Un conjunto de estrategias de defensa únicas}

8 Las primeras investigaciones en psicodinámica del trabajo de mostraron que los trabajadores desarrollan estrategias de defensa que les permiten soportar los aspectos más dolorosos de su actividad (Dejours, 1980). Para cumplir con los requisitos y satisfacer las expectativas de la organización sin enfermarse, los trabajadores utilizan estrategias de defensa contra el sufrimiento, como el individualismo, la negación del peligro o la hiperactividad.

9 Podemos definir estas estrategias como mecanismos mediante los cuales los trabajadores tratan de modificar, transformar y minimizar la percepción de la realidad que los hace sufrir. Estas estrategias buscan una anulación simbólica de peligros reales, sobre todo relacionados con las condiciones de trabajo. Son conductas intencionales y pueden ser individuales o colectivas (en este caso dependen del consenso de un grupo particular de trabajadores y son clave para la construcción de reglas de trabajo), operan a nivel simbólico y tienen un efecto considerable sobre las ideas y los comportamientos de los trabajadores. Todas ellas tienen un impacto en la percepción de la realidad y el conocimiento de los trabajadores: las estrategias defensivas alteran los modos de pensamiento y de expresión. Son estas las estrategias de defensa que permiten que la gran mayoría de los trabajadores se mantengan "normales". Es decir, evitan la descompensación psicopatológica.

10 En el caso del grupo de trabajadores estudiado, esas estrategias toman diferentes formas. Encontramos, por un lado, estrategias de defensa individuales, como la hiperactividad, es decir, una aceleración auto-sostenida y solicitada por los trabajadores. La hiperactividad permite una "anestesia" parcial del pensamiento, gracias a una aplicación extrema en el comportamiento y la motilidad. La atención frenética de llamadas telefónicas y la entrega sin límites a los objetivos de producción permiten a algunos trabajadores ocultarlos conflictos éticos. El mantenimiento de un bullicio comportamental sirve precisamente a evitarla aparición de este tipo de conflictos, repeliéndolos de la conciencia.

11 A nivel colectivo, los trabajadores parecen organizados en diferentes clanes enemigos. La constitución de estos clanes se basa en conflictos de valores y prácticas profesionales, y opone a los trabajadores que consideran la mentira un instrumento profesional legítimo, al servicio de la productividad, con los teleoperadores que prefieren centrarse en un servicio al cliente desprovisto de segundas intenciones. La hostilidad entre los dos grupos así formados es patente, cada uno acusándose mutuamente de ser el origen de las dificultades experimentadas por todos. Las recriminaciones, conflictos y enfrentamientos son frecuentes y pueden incluso conducir a confrontaciones violentas entre colegas. De acuerdo a la psicodinámica del trabajo, enfocarse en los conflictos interpersonales y acusaciones evita un debate constructivo sobre el trabajo real y sus dificultades. Este fenómeno colectivo es característico de lo que llamamos ideologías defensivas, cuya función es, precisamente, evitar cualquier cuestionamiento de las prácticas profesionales. 
12 Además de las estrategias anteriores, es importante mencionar un tercer tipo de estrategias defensivas bastante sorprendentes. Esto es lo que elegimos apodar "estrategia defensiva de regresión infantil". A diferencia de las estrategias de defensa estudiadas tradicionalmente en psicodinámica del trabajo, siempre consideradas creaciones originales y voluntarias de los trabajadores, la estrategia de regresión infantiles promovida-y podríamos decir incluso propuesta- por la administración en forma de juegos y concursos burlescos. Estos concursos o desafíos cumplen una función netamente productiva, y su objetivo es promover la competencia entre los operadores, cuyos criterios de evaluación son la cantidad de ventas. Los equipos o los telemarketers mejor clasificados pueden beneficiar de unos minutos para jugar con una consola de videojuegos o pilotear un helicóptero en miniatura, por ejemplo. Las recompensas y los premios son regularmente de este tipo, lo que muchos trabajadores interpretan como una forma de infantilización. Al parecer, los juegos profesionales incitan a comportamientos infantiles y promueven una forma de regresión o inmadurez intelectual adoptada por gran parte de los vendedores telefónicos. En nuestra opinión, este tipo de comportamiento tiene un aspecto defensivo. La inmadurez intelectual a la que impulsan los juegos se acompaña de una renuncia a la capacidad reflexiva, al servicio de las defensas. Renunciar a un ejercicio pleno e independiente de su intelecto, permite a los teleoperadores evitar la reflexión sobre la naturaleza dudosa de sus prácticas profesionales. Por lo tanto, la regresión infantil sirve para indemnizar a todas y cada una de las consecuencias cotidianas de la mentira, o al menos para evitar cualquier cuestionamiento al respecto.

13 Este conjunto complejo de estrategias defensivas permite que los trabajadores mantengan su equilibrio mental intacto, mediante una dosis de sufrimiento doloroso pero soportable .Sin embargo, este dispositivo informal se despliega en métodos de evaluación que hacen que la capacidad para mentir de manera efectiva se transforme en uno de los criterios de evaluación de la calidad del trabajo.

\section{Reconocimiento y evaluación del trabajo}

14 Las recompensas y premios por desempeño son invariablemente otorgadas a los mejores vendedores del centro de atención telefónica, independientemente de los métodos utilizados para lograr este tipo de actuaciones. Al parecer, quienes son considerados por la dirección como los mejores trabajadores son también quienes utilizan la mentira con más sutileza y menos escrúpulos. Es decir, aquellos para quienes la venta de nuevos productos no implica necesariamente una evaluación de la naturaleza del pedido del cliente, ni de sus necesidades reales. Los mejores vendedores también reciben premios y bonificaciones que constituyen una parte importante de su salario y que los diferencian de los de más colegas. Así, las formas de compensación y reconocimiento del trabajo se encuentran estrechamente relacionadas con los criterios cuantitativos, traducidos principalmente por indicadores numéricos (número de ventas, cantidad de llamadas telefónicas, etc.), pero sin tener en cuenta criterios cualitativos, tales como el respeto por el pedido del cliente o su satisfacción con las respuestas recibidas. Este sistema de evaluación del desempeño conduce lógicamente a consecuencias prácticas que se describen en detalle en la tesis .Por una parte, estos incentivos promueven implícitamente ventas forzadas, abusos comerciales y propagación de mentiras. Por otra parte, la instrumentalización del reconocimiento, 
junto con el sufrimiento ético y las defensas adoptada por los trabajadores, produce mecanismos de sumisión personal tan inesperados como preocupantes.

Los elementos presentados en esta tesis sugieren la necesidad de reevaluarlos actuales modelos de organización del trabajo en centros de atención telefónica, sobre todo si tomamos en cuenta la experiencia subjetiva de los trabajadores. La crítica se basa principalmente en los efectos adversos observados en términos de salud en el trabajo, pero también en consideraciones más amplias relacionadas con la restricción de la autonomía de los trabajadores.

\section{BIBLIOGRAFIA}

Dejours, C. (1998). Souffrance en France. Paris, Seuil.

Dejours, C. (1980). Travail, usure mentale (Ed.2008, revista e ampliada). Paris, Bayard.

Dessors, D. (2009). De l'ergonomie à la psychodynamique du travail. Toulouse, Érès.

Ganem, V. (2011). Relato de uma experiência de terreno de intervenção em Psicodinâmica do Trabalho (PDT). Laboreal, 7, (1), 68-75. http://laboreal.up.pt/revista/artigo.php?

id=48u56oTV 6582235338949985532

Hughes, E.C. (2010). Les honnêtes gens et le sale boulot. Travailler, 24, 21-34.

Wisner, A. (1995). Réflexions sur l'ergonomie. Toulouse, Octarès.

\section{NOTAS}

1. La investigación siempre implica la formación de pequeños grupos de trabajadores, voluntarios, para discutir colectivamente la relación entre las dificultades percibidas y las formas de organización del trabajo. Así, los grupos formados participan en sesiones de trabajo de medio día. Cada grupo se reúne entre 2 y 4 sesiones. El material recogido se presenta entonces a los participantes en la forma de un informe escrito. Este informe será examinado por los participantes y validado por las partes interesadas. Por último, el informe servirá como marco para las discusiones que servirán a la transformación de la organización del trabajo. Los principios metodológicos de la investigación en psicodinámica del trabajo fueron expuestos en detalle por Dejours (1980) y Dessors (2009). 


\section{AUTOR}

\section{DUARTE ROLO}

Equipa de Psicodinâmica do Trabalho e da Ação, Conservatoire National des Arts et Métiers, 41 rue Gay-Lussac, 75005 Paris, Francia

duarte.rolo@cnam.fr 